\title{
THE LOW-DIMENSIONAL HOMOLOGY OF CROSSED MODULES
}

\author{
N. D. GILBERT \\ (communicated by Ronald Brown)
}

\begin{abstract}
This paper is prompted by work of Grandjeán and Ladra on homology crossed modules. We define the homology of a crossed module in dimensions one and two via the equivalence of categories with group objects in groupoids. We show that for any perfect crossed module, its second homology crossed module occurs as the kernel of its universal central extension as defined by Norrie. Our first homology is the same as that defined by Grandjeán and Ladra, but the second homology crossed modules are in general different. However, they coincide for aspherical perfect crossed modules. Our methods can in principle be applied to define a homology crossed module in any dimension.
\end{abstract}

\section{Introduction}

Crossed modules are algebraic models of homotopy 2-types and so are important in homotopical algebra. They are also of algebraic interest as the first stage in "higher dimensional group theory" [2]. To develop this theory, we require definitions of basic group concepts, adapted to the higher dimensional setting and reflecting the extra structure involved, and generalisations of the key group theorems about these concepts. The Ph.D. thesis of K.J. Norrie [19] pursued this idea, concentrating on the theory of automorphism groups, but including analogues of the centre, the commutator subgroup, abelianisation, central extensions, and other related concepts for crossed modules. Norrie's most important results were published in [20]. Recent further development has been carried out by Doncel-Juárez, Grandjeán and Ladra $[6,12,13]$ with emphasis on the low-dimensional homology of crossed modules.

The category of crossed modules is equivalent to the category of group objects in the category of groupoids $[4, \mathbf{1 6}]$, and using this equivalence, we can regard a crossed module as a semidirect product $C$ and apply group constructions to $C$. When the result of some group construction is again equivalent to a crossed module, we have a natural candidate for the construction for crossed modules. This approach may work for non-functorial constructions such as the centre, as well as for functors such as abelianisation, and Norrie's definitions of the centre, the commutator subcrossed module, and abelianisation, all follow from this recipe. However, the analogue of the automorphism group - Norrie's actor crossed module - is not obtained this way. The full automorphism group $\operatorname{Aut}(C)$ does not necessarily respect all the extra structure coming from a crossed module, and Norrie demonstrated that a subgroup $\mathcal{A}$ of $\operatorname{Aut}(C)$ identified by Lue $[\mathbf{1 7}]$ was the appropriate "automorphism object". A categorytheoretic interpretation of $\mathcal{A}$ was given in [10].

Grandjeán and Ladra $[\mathbf{1 2}, \mathbf{1 3}]$ have extended Norrie's work on central extensions for crossed modules and have defined the second homology crossed module by means of a Hopf formula

I am grateful to W.A. Bogley for several discussions on the ideas in this paper, and to R.Brown and J. Howie for their valuable comments. I am indebted to the referee for drawing my attention to the work of Carrasco, Cegarra and Grandjeán.

Received 12 January 2000, revised 1 June 2000; published on 7 June 2000.

2000 Mathematics Subject Classification: 20J05, 20L10, 18D35, 18G50.

Key words and phrases: homology, crossed module, groupoid.

(C) 2000, N. D. Gilbert. Permission to copy for private use granted. 
applied to a projective presentation of a crossed module. However, the direct functorial approach is available: the homology group $H_{2}(C)$ is equivalent to an abelian crossed module that is an alternative candidate for the second homology crossed module. In this paper we investigate the properties of this candidate and its relationship to the Grandjeán-Ladra construction. We show that our second homology occurs as the kernel of Norrie's universal central extension for perfect crossed modules, and coincides with the Grandjeán-Ladra construction when applied to an aspherical perfect crossed module. Finally we relate the second homology to crossed module descriptions of relative algebraic $K$-theory.

An alternative approach to the homology and cohomology of crossed modules has been developed by Carrasco, Cegarra and Grandjeán [5]. They successfully generalise the BarrBeck cotriple homology and cohomolgy of groups [1] to crossed modules, based on a tripleable underlying functor to the category of sets. The Carrasco, Cegarra and Grandjeán second homology crossed module again coincides with the Grandjeán-Ladra construction (and so with the second homology developed in the present paper) for aspherical perfect crossed modules. It would be interesting to know the precise relationships between these three constructions: we make some remarks on this in section 4 .

\subsection{Remark on notation}

Our chosen convention for a semidirect product with $G$ acting on $T$ on the right gives $T \rtimes G$ the composition $(s, g)(t, h)=\left(s^{h} t, g h\right), s, t \in T, g, h \in G$. In particular, $(s, g)=(1, g)(s, 1)$.

\section{Low-dimensional homology for crossed modules}

A crossed module $(T, G, \partial)$ consists of a group homomorphism $\partial: T \rightarrow G$ together with a group action of $G$ on $T$ satisfying, for all $s, t \in T$ and $g \in G$,

$$
\begin{aligned}
\partial\left(t^{g}\right) & =g^{-1} \partial(t) g, \\
t^{\partial(s)} & =s^{-1} t s .
\end{aligned}
$$

The survey $[\mathbf{2}]$ is a good reference for the theory of crossed modules and their applications in homotopy theory.

There is an equivalence between the category of crossed modules and the category of group objects in the category of groupoids $[\mathbf{4}]$ (see also [16]). A crossed module $(T, G, \partial)$ is equivalent to the group $C=T \rtimes G$ carrying the groupoid structure with source and target maps

$$
\begin{aligned}
& \sigma: T \rtimes G \rightarrow G ; \sigma(t, g)=g \partial(t), \\
& \tau: T \rtimes G \rightarrow G ; \tau(t, g)=g
\end{aligned}
$$

and with composition

$$
(s, h) \cdot(t, g)=(t s, g)
$$

defined whenever $h=g \partial(t)$.

The centre $Z(C)=T^{G} \rtimes\left(\operatorname{stab}_{G}(T) \cap Z(G)\right)$ is equivalent to the crossed module

$$
\left.\partial\right|_{T^{G}}: T^{G} \rightarrow \operatorname{stab}_{G}(T) \cap Z(G),
$$

which is Norrie's definition of the centre, and likewise the commutator subgroup of $C,[C, C]=$ $[T, G] \rtimes[G, G]$ is equivalent to the crossed module

$$
\left.\partial\right|_{[T, G]}:[T, G] \rightarrow[G, G],
$$

which is Norrie's definition of the commutator subcrossed module. The abelianisation of $C$,

$$
H_{1}(C)=C^{a b}=T_{G} \times G^{a b}
$$

is equivalent to the crossed module

$$
\bar{\partial}: T_{G} \rightarrow G^{a b},
$$


in which $\bar{\partial}$ is induced by $\partial$ and $G^{a b}$ acts trivially on $T_{G}$. So we obtain the abelianised, or first homology crossed module

$$
H_{1}(T, G, \partial)=\left(T_{G}, G^{a b}, \bar{\partial}\right) .
$$

This coincides with the definition of $H_{1}(T, G, \partial)$ given in [19] and [13]. To define the second homology crossed module, we proceed in the same way.

Proposition 2.1. The second homology group $H_{2}(C)$ is equivalent to a crossed module $\sigma_{*}$ : $\Sigma \rightarrow H_{2}(G)$ of abelian groups, where $\sigma_{*}$ is induced by $\sigma$, and $\Sigma$ fits into an exact sequence

$$
H_{2}\left(G, H_{1}(T)\right) \rightarrow H_{2}(T)_{G} \rightarrow \Sigma \rightarrow H_{1}\left(G, H_{1}(T)\right) \rightarrow 0 .
$$

Proof. The exact sequence for $\Sigma$ is a standard result obtained from the Lyndon-HochschildSerre spectral sequence for $H_{2}(C)=H_{2}(T \rtimes G)$, where $H_{2}(C)$ splits as $\Sigma \oplus H_{2}(G)$. Now $H_{2}(C)$ carries a groupoid structure with source and target maps $\sigma_{*}, \tau_{*}: H_{2}(C) \rightarrow H_{2}(G)$ induced by $\sigma$ and $\tau$. The equivalent crossed module is $\sigma_{*}: \operatorname{ker} \tau_{*} \rightarrow H_{2}(G)$, and since $\tau_{*}$ is the projection to $H_{2}(G)$ we see that $\operatorname{ker} \tau_{*}=\Sigma$.

We denote the second homology crossed module identified in proposition 2.1 by $\mathcal{H}_{2}(T, G, \partial)$, to distinguish it from the Grandjeán-Ladra version, which we shall discuss below and denote by $H_{2}^{G L}(T, G, \partial)$.

\subsection{Examples}

(a) A group $G$ may be regarded as a crossed module in two ways, as the inclusion $i: 1 \rightarrow$ $G$ of the trivial subgroup into $G$, and as the identity map id : $G \rightarrow G$. In the first case, $\mathcal{H}_{2}(1, G, i)=\left(0, H_{2}(G), i\right)$. For the second case, we may use the isomorphism $G \times G \rightarrow G \rtimes G$ given by $(g, h) \mapsto\left(g^{-1} h, g\right)$. Under composition with this isomorphism, $\sigma$ and $\tau$ become the projections from $G \times G$ to $G$. Hence

$$
\mathcal{H}_{2}(G, G, \mathrm{id})=\left(H_{2}(G) \oplus\left(H_{1}(G) \otimes H_{1}(G)\right), H_{2}(G), \sigma_{*}\right),
$$

with $\sigma_{*}$ acting as the identity map on $H_{2}(G)$ and as the zero map on $H_{1}(G) \otimes H_{1}(G)$.

(b) An abelian group $A$ forms a crossed module $(A, 1,0)$, and we have $\mathcal{H}_{2}(A, 1,0)=$ $\left(H_{2}(A), 0,0\right)$. This contrasts with $H_{2}^{G L}(A, 1,0)$ which is always trivial [13].

(c) A $G$-module $M$ gives a crossed module $(M, G, 0)$, for which $\sigma=\tau:(m, g) \mapsto g$. Hence $\left.\sigma_{*}\right|_{\text {ker } \tau_{*}}$ is trivial, and $\mathcal{H}_{2}(M, G, 0)=\left(\Sigma, H_{2}(G), 0\right)$.

(d) In any central extension $0 \rightarrow N \rightarrow E \stackrel{\pi}{\rightarrow} Q \rightarrow 1$ of groups, $(E, Q, \pi)$ is a crossed module. If $Q$ is perfect and $E=\tilde{Q}$ is its universal central extension (see [18], for example) then $H_{1}(\tilde{Q})=H_{2}(\tilde{Q})=0$ and proposition $(2.1)$ shows that $\Sigma=0$. Hence $\mathcal{H}_{2}(\tilde{Q}, Q, \pi)=$ $\left(0, H_{2}(Q), i\right)$.

(e) Even for free crossed modules over free groups, $\mathcal{H}_{2}$ may be non-trivial. Let $F$ be an arbitrary non-abelian free group, and let $Y$ be the free crossed $F$-module with a single basis element $y \in F$, with $G=F / \ll y \gg$. If $y$ is not a proper power in $F$ then $H_{2}(Y)=0$, and by Lyndon's Identity Theorem, $H_{1}(Y)$ is the free $\mathbb{Z} G$-module with basis $\{y\}$. From (2.1) we see that $\Sigma \cong H_{1}\left(F, H_{1}(Y)\right) \cong H_{1}(F, \mathbb{Z} G) \cong H_{1}(Y, \mathbb{Z})$.

(f) If $G$ is a classical knot group, then by Theorem 2.2 of [21], id : $G \rightarrow G$ is a free crossed module with a one-element basis. From example (a), we have in this case $\mathcal{H}_{2}(G, G$, id) $=$ $(\mathbb{Z}, 0,0)$.

(g) By Theorem 4.1 of $[\mathbf{2 1}]$, a superperfect normal subgroup $N$ of a group $G$ gives a projective crossed module $(N, G, i)$ that is not free. In this case we have $\mathcal{H}_{2}(N, G, i)=\left(0, H_{2}(G), i\right)$.

Remark 2.2. Ellis [9] defines the (co)homology groups of a crossed module $(T, G, \partial)$ as the (co)homology of its classifying space $B(T, G, \partial)$ (see [2] for an account of the classifying space). Ellis develops a number of interesting properties of $H_{*} B(T, G, \partial)$, showing for example that $H_{1}(B(T, G, \partial))=H_{1}($ coker $\partial)$, and that $H_{*}(B(1, G, i))=H_{*}(G)$. However since $B(G, G$, id $)$ 
is contractible, $H_{2}(B(G, G, \mathrm{id}))$ is always trivial, in contrast to the crossed module homology $\mathcal{H}_{2}\left(G, G\right.$, id) (and to $H_{2}^{G L}(T, G, \partial)$ ).

\section{The Grandjeán-Ladra homology}

The Grandjeán-Ladra second homology $H_{2}^{G L}(T, G, \partial)$ of a crossed module $(T, G, \partial)$ is a crossed module $\left(J, H_{2}(G), \mu_{*}\right)$, where $J$ is an abelian group given by a Hopf-type formula. More precisely, if $(T, G, \partial)$ is presented by an $\mathcal{E}$-projective presentation

$$
(V, R, \mu) \rightarrow(Y, F, \mu) \rightarrow(T, G, \partial)
$$

(see $[\mathbf{1 2}]$ for the notion of $\mathcal{E}$-projectives) then

$$
J=\frac{V \cap[Y, F]}{[Y, R][V, F]}
$$

with the map

$$
\mu_{*}: \frac{V \cap[Y, F]}{[Y, R][V, F]} \rightarrow \frac{R \cap[F, F]}{[R, F]}
$$

induced by $\mu$.

We replace the $\mathcal{E}$-projective presentation above by the equivalent group extension

$$
V \rtimes R \rightarrow Y \rtimes F \rightarrow T \rtimes G
$$

and consider the 5-term homology sequence (writing $V R$ for $V \rtimes R$ etc.)

$$
H_{2}(Y \rtimes F) \rightarrow H_{2}(T \rtimes G) \rightarrow \frac{V R}{[V R, Y F]} \rightarrow H_{1}(Y \rtimes F) \rightarrow H_{1}(T \rtimes G) \rightarrow 0 .
$$

Each term splits: $H_{2}(T \rtimes G)=\Sigma \oplus H_{2}(G)$ as above, and likewise $H_{2}\left((Y \rtimes F)=B \oplus H_{2}(F)\right.$ for some abelian group $B$, whilst $H_{1}(T \rtimes G)=T_{G} \oplus H_{1}(G)$ and $H_{1}\left((Y \rtimes F)=Y_{F} \oplus H_{1}(F)\right.$. Finally,

$$
\begin{aligned}
\frac{V R}{[V R, Y F]} & =\frac{V R}{[V, Y][V, F][R, Y][R, F]} \\
& =\frac{V R}{[V, F][R, Y][R, F]}(\text { since }[V, Y] \subseteq[R, Y]) \\
& =\frac{V}{[V, F][R, Y]} \oplus \frac{R}{[R, F]},
\end{aligned}
$$

this last splitting arising since

$$
\begin{aligned}
R \cap[V, Y][R, Y][R, F] & =(R \cap[V, Y][R, Y])[R, F] \text { (modular law) } \\
& =[R, F] .
\end{aligned}
$$

The 5-term homology sequence then splits into the direct sum of the sequences

$$
H_{2}(F) \rightarrow H_{2}(G) \rightarrow \frac{R}{[R, F]} \rightarrow H_{1}(F) \rightarrow H_{1}(G) \rightarrow 0
$$

(the standard 5-term sequence for $G$ presented as $F / R$ ) and

$$
B \rightarrow \Sigma \rightarrow \frac{V}{[V, F][R, Y]} \rightarrow Y_{F} \rightarrow T_{G} \rightarrow 0 .
$$

Proposition 3.1. Let $(T, G, \partial)$ be a crossed module and consider the second homology crossed modules $\mathcal{H}_{2}(T, G, \partial)=\left(\Sigma, H_{2}(G), \sigma_{*}\right)$ and $H_{2}^{G L}(T, G, \partial)=\left(J, H_{2}(G), \mu_{*}\right)$, the latter defined by an $\mathcal{E}$-projective presentation

$$
(V, R, \mu) \rightarrow(Y, F, \mu) \rightarrow(T, G, \partial) .
$$


If $H_{2}(Y \rtimes F)=B \oplus H_{2}(F)$ then there exists an exact sequence of abelian groups

$$
B \rightarrow \Sigma \rightarrow J \rightarrow 0 .
$$

Moreover, we may assume that $B$ fits into a short exact sequence

$$
0 \rightarrow H_{2}(Y)_{F} \rightarrow B \rightarrow H_{1}\left(F, H_{1}(Y)\right) \rightarrow 0,
$$

with $F$ a free group and $Y$ a free crossed $F$-module.

Proof. The exact sequence $B \rightarrow \Sigma \rightarrow J \rightarrow 0$ follows from the definition of $J$ and the sequence (3.2). Since $H_{2}^{G L}(T, G, \partial)$ is independent of the chosen $\mathcal{E}$-projective presentation [12] we may

take $F$ to be a free group mapping to $G$ and $Y$ to be a free crossed $F$-module. Then the short exact sequence for $B$ follows from the Lyndon-Hochschild-Serre spectral sequence for the split extension $Y \rtimes F$.

\section{Universal central extensions}

One of the achievements of [19] was the identification of the universal central extension of a crossed module in terms of the Brown-Loday non-abelian tensor product, generalising the description given by Brown and Loday for the group case [3]. Given two groups $M$ and $N$ acting upon one another (on the right), their tensor product is the group generated by symbols $m \otimes n, m \in M, n \in N$ subject to relations (in the modified form given in [11]):

$$
\begin{aligned}
m m_{0} \otimes n & =\left(m_{0} \otimes n^{m}\right)(m \otimes n), \\
m \otimes n n_{0} & =(m \otimes n)\left(m^{n} \otimes n_{0}\right) .
\end{aligned}
$$

If $M$ and $N$ are crossed $P$-modules acting on one another via $P$, then they also act on $M \otimes N$ by

$$
(m \otimes n)^{m_{1}}=m_{1}^{-1} m m_{1} \otimes n^{m_{1}}, \quad(m \otimes n)^{n_{1}}=m^{n_{1}} \otimes n_{1}^{-1} n n_{1} .
$$

Given a perfect crossed module $(T, G, \partial)$, that is one that satisfies $H_{1}(T, G, \partial)=(1,1, i)$, Norrie [19] shows that the crossed module $(T \otimes G, G \otimes G, \partial \otimes$ id) is the universal central extension of $(T, G, \partial)$, where $G$ acts on itself by conjugation, and $G \otimes G$ acts on $T \otimes G$ via the commutator map $G \otimes G \rightarrow G, g \otimes h \mapsto g^{-1} h^{-1} g h$.

We can also obtain a tensor product formulation of $\mathcal{H}_{2}(T, G, \partial)$ by using a truncated version of the 8-term homology sequence from [3]. If $N$ is a normal subgroup of $P$ such that $N=$ $[N, P]$, and if $Q=P / N$, there is an exact sequence

$$
H_{3}(P) \rightarrow H_{3}(Q) \rightarrow \operatorname{ker}(\kappa: N \otimes P \rightarrow N) \rightarrow H_{2}(P) \rightarrow H_{2}(Q) \rightarrow 0
$$

where $\kappa: N \otimes P \rightarrow N$ by $n \otimes p \mapsto[n, p]$. Note that following [3], we expect to see a nonabelian exterior product $N \wedge P$ in the third term. However, Theorem 2.12 of [3] shows that if $N=[N, P]$ then $N \wedge P=N \otimes P$. Moreover, the right-hand 0 arises from truncation of the sequence at the term $N /[N, P]=0$. If in addition we have a splitting $Q \rightarrow P$, so that $P \cong N \rtimes Q$ and $H_{2}(P)=A \oplus H_{2}(Q)$, then we obtain an isomorphism $A \cong \operatorname{ker}(N \otimes P \rightarrow N)$. Note that if we take $N=T \triangleleft T \rtimes G=P$, then $\kappa: s \otimes(t, g) \mapsto[(s, 1),(t, g)]=\left(s^{-1} t^{-1} s^{g} t, 1\right) \in T$.

Proposition 4.1. If $(T, G, \partial)$ is a perfect crossed module with second homology crossed module $\mathcal{H}_{2}(T, G, \partial)=\left(\Sigma, H_{2}(G), \sigma_{*}\right)$, then there exists an isomorphism

$$
\Sigma \cong \operatorname{ker}(T \otimes(T \rtimes G) \rightarrow T) .
$$

In order to relate $\mathcal{H}_{2}(T, G, \partial)$ to Norrie's universal central extension of crossed modules, we need to understand the tensor product $T \otimes(T \rtimes G)$.

Proposition 4.2. For any crossed module $(T, G, \partial)$, the tensor product $T \otimes(T \rtimes G)$ has an abelian normal subgroup $\mathcal{K}$ such that $T \otimes(T \rtimes G)$ splits as the direct product

$$
T \otimes(T \rtimes G) \cong \mathcal{K} \times(T \otimes G) .
$$


Proof. A check on the preservation of defining relations shows that the mapping

$$
\operatorname{id} \otimes \sigma: s \otimes(t, g) \mapsto s \otimes g \partial(t)
$$

defines a homomorphism $T \otimes(T \rtimes G) \rightarrow T \otimes G$ which is split by the map $\nu: s \otimes g \mapsto s \otimes(1, g)$. Set $\mathcal{K}=\operatorname{ker}($ id $\otimes \sigma)$. Now if $\kappa^{\prime}: T \otimes G \rightarrow T$ maps $t \otimes g \mapsto t^{-1} t^{g}$, we have $\kappa^{\prime} \circ($ id $\otimes \sigma)=\kappa$, and Proposition 2.3 of $[\mathbf{3}]$ shows that $(T \otimes(T \rtimes G), T, \kappa)$ is a crossed module and so has central kernel. Since $\mathcal{K} \subseteq \operatorname{ker} \kappa$, the proposition follows.

\subsection{Examples}

(a) Let $A$ be an abelian group and set $T=A=G$ with $\partial=$ id and $A$ acting trivially on itself. Then $\mathcal{K} \cong A \oplus A$.

(b) Let $M$ be a $G$-module, and let $C=M \rtimes G$. Then $M$ is also a $C$-module, with $M$ acting trivially on itself. Guin [14] shows that $M \otimes C \cong M \otimes_{\mathbb{Z} C} \mathfrak{c}$ (where $\mathfrak{c}$ is the augmentation ideal of $\mathbb{Z} C$ ), and under this isomorphism, the map id $\otimes \sigma$ becomes a map $M \otimes_{\mathbb{Z} C} \mathfrak{c} \rightarrow M \otimes_{\mathbb{Z} G} \mathfrak{g}$ given by $m \otimes(1-(n, g)) \mapsto m \otimes(1-g)$.

Theorem 4.3. If $(T, G, \partial)$ is a perfect crossed module, then the subgroup $\mathcal{K}$ of $T \otimes(T \rtimes G)$ is trivial, and the map

$$
\operatorname{id} \otimes \sigma: T \otimes(T \rtimes G) \rightarrow T \otimes G
$$

is an isomorphism.

Proof. We first show that the splitting $\nu: T \otimes G \rightarrow T \otimes(T \rtimes G)$ from the proof of Proposition 4.2 is $T$-equivariant. Since $(T, G, \partial)$ is a perfect crossed module, the map $\kappa^{\prime}: T \otimes G \rightarrow T$ is surjective, and so given $s \in T$ we may write $s=\kappa^{\prime}(y)$ for some $y \in T \otimes G$. Since $\left(T \otimes G, T, \kappa^{\prime}\right)$ is a crossed module, we have, for all $t \in T, g \in G$ that

$$
(t \otimes g)^{s}=(t \otimes g)^{\kappa^{\prime}(y)}=y^{-1}(t \otimes g) y .
$$

Now $\kappa \circ \nu=\kappa^{\prime}$ and it follows that

$$
\begin{aligned}
\nu\left((t \otimes g)^{s}\right) & =\nu\left(y^{-1}(t \otimes g) y\right)=\nu\left(y^{-1}\right) \nu(t \otimes g) \nu(y) \\
& =\nu(t \otimes g)^{\kappa(\nu(y))}=\nu(t \otimes g)^{\kappa^{\prime}(y)} \\
& =\nu(t \otimes g)^{s} .
\end{aligned}
$$

We now claim that $\nu$ is surjective. In fact we shall show that in $T \otimes(T \rtimes G)$ we have, for all $s, t \in T$ and $g \in G$,

$$
s \otimes(t, g)=s \otimes(1, g \partial(t))
$$

Rewriting (4.1) as

$$
s \otimes(1, g)(t, 1)=s \otimes(1, g)(1, \partial(t))
$$

and expanding both sides, we see that (4.1) is equivalent to

$$
s \otimes(t, 1)=s \otimes(1, \partial(t))
$$

for all $s, t \in T$. Since $T$ is generated by elements of the form $u^{-1} u^{g},(u \in T, g \in G)$, it suffices to establish (4.2) for $t=u^{-1} u^{g}$, so that $\partial(t)=[\partial(u), g]$.

We let $\lambda: T \otimes G \rightarrow G$ be the map defined by $t \otimes g \mapsto[\partial(t), g]$. By Proposition 2.3 of $[\mathbf{3}]$, $(T \otimes G, G, \lambda)$ is a crossed module. Moreover, again using Proposition 2.3 of [3], we have (with due allowance for the change of notation from the left actions in $[\mathbf{3}]$ ),

$$
s \otimes\left(u^{-1} u^{g}, 1\right)=s \otimes \kappa(u \otimes(1, g))=(u \otimes(1, g))^{-s}(u \otimes(1, g)) .
$$


whereas

$$
\begin{aligned}
s \otimes(1,[\partial(u), g]) & =\nu(s \otimes[\partial(u), g]) \\
& =\nu(s \otimes \lambda(u \otimes g)) \\
& =\nu\left((u \otimes g)^{-s}(u \otimes g)\right) \\
& =\nu\left((u \otimes g)^{-s}\right) \nu(u \otimes g) \\
& =(\nu(u \otimes g))^{-s} \nu(u \otimes g) \quad \text { (by } T \text {-equivariance) } \\
& =(u \otimes(1, g))^{-s}(u \otimes(1, g)) .
\end{aligned}
$$

Comparing (4.3) and (4.4) completes the proof.

Remark 4.4. Since $\mathcal{K}$ is generated by all elements $s \otimes\left(t^{-1}, \partial(t)\right)$, the proof of Theorem 4.3 also shows directly that $\mathcal{K}$ is trivial when $(T, G, \partial)$ is perfect. Moreover, the proof is valid if we assume only that $T$ is $G$-perfect, that is $T_{G}=1$.

Proposition 4.1 and Theorem 4.3 combine to show that the kernel of Norrie's universal central extension of a perfect crossed module is the second homology $\mathcal{H}_{2}$ of that crossed module:

Corollary 4.5. [19] If $(T, G, \partial)$ is a perfect crossed module, then the central extension

$$
0 \rightarrow \mathcal{H}_{2}(T, G, \partial) \rightarrow(T \otimes G, G \otimes G, \partial \otimes \mathrm{id}) \rightarrow(T, G, \partial) \rightarrow 1
$$

is universal.

A crossed module $(T, G, \partial)$ is aspherical if $\partial$ is injective. Grandjeán and Ladra [13] show that for an aspherical, perfect crossed module $(T, G, \partial)$, in the notation of section 3 , the central extension

$$
0 \rightarrow H_{2}^{G L}(T, G, \partial) \rightarrow\left(\frac{[F, Y]}{[R, Y][F, V]}, \frac{[F, F]}{[R, F]}, \mu_{*}\right) \rightarrow(T, G, \partial) \rightarrow 1
$$

is universal. As a corollary, they deduce that for an aspherical, perfect crossed module $(T, G, \partial)$ there is an isomorphism

$$
T \otimes G \cong \frac{[F, Y]}{[R, Y][F, V]} .
$$

For the second homology we have the following consequence of the universal property of (4.5).

Corollary 4.6. If $(T, G, \partial)$ is an aspherical, perfect crossed module then $H_{2}^{G L}(T, G, \partial)$ is isomorphic to $\mathcal{H}_{2}(T, G, \partial)$. In particular the groups $J$ and $\Sigma$ are isomorphic.

Remark 4.7. A far-reaching general approach to the homology and cohomology of crossed modules has been developed recently by Carrasco, Cegarra and Grandjeán [5]. They define a cotriple homology and cohomology theory based on the underlying functor from crossed modules to sets that takes a crossed module $(T, G, \partial)$ to the set $T \times G$. The result, analogous to the Barr-Beck theory for groups [1], yields homology crossed modules and cohomology groups for crossed modules.

The homology crossed module $H_{n}^{\bullet}(T, G, \partial),(n \geqslant 1)$ defined by Carrasco, Cegarra and Grandjeán is an abelian crossed module of the form $Z_{n} \rightarrow H_{n}(G)$ for some abelian group $Z_{n}$. For $n=1, H_{1}^{\bullet}(T, G, \partial)$ is again the abelianised crossed module $\left(T_{G}, G^{a b}, \bar{\partial}\right)$. For $n=2$, it is shown in [5] that for a perfect crossed module $(T, G, \partial)$, the homology group $H_{2}^{\bullet}(T, G, \partial)$ is the kernel of the universal central extension of $(T, G, \partial)$, and it follows that if $(T, G, \partial)$ is perfect then $H_{2}^{\bullet}(T, G, \partial)=\mathcal{H}_{2}(T, G, \partial)$.

However, the constructions do not always coincide. By proposition 8(iv) of [5], for a projective crossed module $(T, G, \partial)$, the homology crossed module $H_{2}^{\bullet}(T, G, \partial)$ is zero. However, if 
$F=F(x, y)$ is a free group of rank 2 and if $N$ is the normal closure in $F$ of $\{x\}$, then $(N, F, i)$ is free (relative to the underlying functor from crossed modules to sets) but as in Example $2.1(\mathrm{e}), \mathcal{H}_{2}(N, F, i)$ is non-zero in general.

Since a computation of the Barr-Beck homology of the semidirect product $T \rtimes G$ involves the underlying functor $T \rtimes G \rightarrow T \times G$ from groups to sets, there is a direct connection between the Carrasco, Cegarra and Grandjeán approach and that in the present paper. It would be very interesting to clarify the relationships beween the results of the two approaches.

Remark 4.8. Using the Brown-Loday non-abelian tensor product, Guin [14] studied the low dimensional homology of a group $G$ with coefficients in a crossed $G$-module $T$. He defined

$$
H_{0}(G, T)=T_{G}, H_{1}(G, T)=\operatorname{ker}\left(\kappa^{\prime}: T \otimes G \rightarrow T\right) .
$$

Hence we have an isomorphism of crossed modules

$$
\mathcal{H}_{1}(T, G, \partial) \cong\left(H_{0}(G, T), H_{1}(G), \bar{\partial}\right)
$$

and if $(T, G, \partial)$ is perfect,

$$
\mathcal{H}_{2}(T, G, \partial) \cong\left(H_{1}(G, T), H_{2}(G), \sigma_{*}\right) .
$$

It would be interesting to pursue higher-dimensional versions of these isomorphisms.

\section{An application to relative algebraic $K$-theory}

It is well known that universal central extensions can be used to frame the definition of $K_{2}$ of a ring $R$. Let $E(R)$ be the subgroup of $G L(R)$ generated by the elementary matrices: the Steinberg group $S t(R)$ is the universal central extension of $E(R)$ and Milnor [18] defined $K_{2}(R)$ as the kernel of $\phi: S t(R) \rightarrow E(R)$. It follows that $K_{2}(R)$ can be identified with the second homology group $H_{2}(E(R))$. Now given a two-sided ideal $I$ of $R$, there are relative groups $K_{i}(R, I), i \geqslant 1$, most easily defined by setting $K_{i}(R, I)=\pi_{i} K(R, I)$ where $K(R, I)$ is the homotopy fibre of $B G L(R)^{+} \rightarrow B G L(R / I)^{+}$. Loday [15] studied $K_{2}(R, I)$ by the construction of a relative universal central extension of the pair $(S t(R), S t(R / I)$ using crossed modules. We show that universal central extensions of crossed modules provide a direct extension of the definition of $K_{2}$ from the absolute to the relative case, amplifying a remark made by Ellis in $[7]$.

Let $F(R)$ denote the homotopy fibre of $B G L(R) \rightarrow B G L(R)^{+}$so that $S t(R) \cong \pi_{1} F(R)$, and let $G L(R, I)$ be the kernel of $G L(R) \rightarrow G L(R / I)$, with $E(I)=E(R) \cap G L(R, I)$. Let $F(R, I)$ denote the homotopy fibre of $F(R) \rightarrow F(R / I)$ : it is homotopy equivalent to the homotopy fibre of $B G L(R, I) \rightarrow K(R, I)$. The relative Steinberg group $S t(R, I)$ may be defined by $\operatorname{St}(R, I)=\pi_{1} F(R, I)$ and the homotopy sequence of the fibration $F(R, I) \rightarrow B G L(R, I) \rightarrow$ $K(R, I)$ translates to the sequence

$$
0 \rightarrow K_{2}(R, I) \rightarrow S t(R, I) \rightarrow G L(R, I) \rightarrow K_{1}(R, I) \rightarrow 0
$$

[15]. We show how $K_{2}(R, I)$ arises from a universal central extension of a crossed module.

It follows from well known relations between elementary matrices that $E(I)=[E(I), E(R)]$ so that $i: E(I) \hookrightarrow E(R)$ is a perfect crossed module. Its universal central extension is the crossed module $E(I) \otimes E(R) \rightarrow E(R) \otimes E(R) \quad$ : now $E(R) \otimes E(R)$ is the universal central extension of $E(R)[\mathbf{3}]$ so that $E(R) \otimes E(R) \cong S t(R)$, and the kernel of $E(R) \otimes E(R) \rightarrow E(R)$ can therefore be identified with $K_{2}(R)$. That a corresponding identification can be made in the relative case follows from a theorem of Ellis. Theorem 1.2 of [8] specialises to an isomorphism $S t(R, I) \cong E(I) \otimes E(R)$ and it follows that $K_{2}(R, I)$ can be identified with the kernel of $E(I) \otimes E(R) \rightarrow E(R)$. To summarise, we have the following result. 
Theorem 5.1. The crossed module $E(I) \hookrightarrow E(R)$ is perfect and the short exact sequence

$$
\begin{aligned}
& 0 \rightarrow K_{2}(R, I) \rightarrow S t(R, I) \rightarrow E(I) \rightarrow 1 \\
& 0 \rightarrow \underset{K_{2}(R)}{\rightarrow} \rightarrow \operatorname{St}(R) \rightarrow E(R) \rightarrow 1
\end{aligned}
$$

is a universal central extension of crossed modules. Hence the crossed module $K_{2}(R, I) \rightarrow$ $K_{2}(R)$ is isomorphic to the second homology crossed module $\mathcal{H}_{2}(E(I), E(R), i)$ (which is equal to $H_{2}^{G L}(E(I), E(R), i)$ since $(E(I), E(R), i)$ is aspherical and perfect).

\section{References}

[1] M. Barr and J. Beck, Homology and standard constructions. In Seminar on triples and categorical homology theory, Lect. Notes in Math 80, 245-335, Springer Verlag (1969).

[2] R. Brown, Groupoids and crossed objects in algebraic topology. Homology, Homotopy and Appl. 1 (1999) 1-78.

[3] R. Brown and J.-L. Loday, Van Kampen theorems for diagrams of spaces. Topology 26 (1987) 311-335.

[4] R. Brown and C.B. Spencer, $G$-groupoids, crossed modules, and the fundamental group of a topological group. Proc. Kon. Ned. Acad. v. Wet. 79 (1976) 296-302.

[5] P. Carrasco, A.M. Cegarra and A.R. Grandjeán, (Co)Homology of crossed modules. Elsevier Preprint (1999).

[6] J.L. Doncel-Juárez and A.R. Grandjeán, q-perfect crossed modules. J. Pure Appl. Algebra 81 (1992) 279-292.

[7] G.J. Ellis, An eight-term exact sequence in algebraic K-theory. Bull. London Math. Soc. 20 (1988) 245-247.

[8] G.J. Ellis, Multirelative algebraic K-theory: the group $K_{2}\left(\Lambda ; I_{1}, \ldots, I_{n}\right)$ and related computations. J. Algebra 112 (1988) 271-289.

[9] G.J. Ellis, Homology of 2-types. J. London Math. Soc. (2) 46 (1992) 1-27.

[10] N.D. Gilbert, Derivations, automorphisms and crossed modules. Comm. Algebra 18 (1990), 2703-2734.

[11] N.D. Gilbert and P.J. Higgins, The non-abelian tensor product of groups and related constructions. Glasgow Math. J. 31 (1989) 17-29.

[12] A.R. Grandjeán and M. Ladra, Crossed modules and homology. J. Pure Appl. Algebra 95 (1994) 41-55.

[13] A.R. Grandjeán and M. Ladra, $H_{2}(T, G, \partial)$ and central extensions for crossed modules. Proc. Edinburgh Math. Soc. 42 (1999) 169-177.

[14] D. Guin, Homologie et cohomologie non-abelienne de groupes. J. Pure Appl. Algebra 50 (1988) 109-137.

[15] J.-L. Loday, Cohomologie et groupes de Steinberg relatifs. J. Algebra 54 (1978) 178-202.

[16] J.-L. Loday, Spaces with finitely many non-trivial homotopy groups. J. Pure Appl. Algebra 24 (1982) 179-202.

[17] A.S-T. Lue, Semicomplete crossed modules and holomorphs of groups. Bull. London Math. Soc. 11 (1979) 8-16.

[18] J. Milnor, Introduction to Algebraic K-theory. Ann. Math. Studies 72, Princeton Univ. Press (1971).

[19] K.J. Norrie, Crossed modules and analogues of group theorems. Ph.D. Thesis, University of London (1987). 
[20] K.J. Norrie, Actions and automorphisms of crossed modules. Bull. Soc. Math. France 118 (1990) 129-146.

[21] J.G. Ratcliffe, Free and projective crossed modules. J. London Math. Soc (2) 22 (1980) 66-74.

This article may be accessed via WWW at http://www.rmi.acnet.ge/hha/ or by anonymous ftp at ftp://ftp.rmi.acnet.ge/pub/hha/volumes/2000/n4/n4.(dvi,ps,dvi.gz,ps.gz)

N. D. Gilbert N.D.Gilbert@ma.hw.ac.uk

Department of Mathematics

Heriot-Watt University

Riccarton, Edinburgh EH14 4AS

Scotland 